\title{
Dydrogesterone has no effect on uterine fibroids when used to prevent miscarriage in pregnant women with uterine fibroids
}

\author{
Shan Wang ${ }^{1}$, Xie-Tong Wang ${ }^{1}$, Rui-Hua Liu ${ }^{2}$, Ming Liu ${ }^{1}$, Yu-Rui Wu ${ }^{1}$, \\ Xiao-Yan Huang ${ }^{1}$, Na Zhao ${ }^{3}$, Yu-Lan $\mathrm{Mu}^{1}$ \\ ${ }^{1}$ Department of Obstetrics and Gynecology, Shandong Provincial Hospital Affiliated to Shandong University, Jinan, China \\ ${ }^{2}$ Ultrasound room, Yantai Yuhuangding Hospital, Yantai, Shandong Province, China \\ ${ }^{3}$ Department of Human Anatomy and Histology \& Embryology, Cheeloo College of Medicine, Shandong University, Jinan, China
}

\begin{abstract}
Objectives: To analyse the effect of dydrogesterone use during pregnancy on uterine fibroids, pregnancy complications, and pregnancy outcome.

Material and methods: In all, 372 pregnant women with uterine fibroids who were treated at the Affiliated Provincial Hospital of Shandong University were included in this study. Thirty-three of these women received dydrogesterone and constituted the treatment group, and the 27 women who were found to have uterine fibroids during the first trimester but did not receive intervention to prevent miscarriage composed the control group. The changes in uterine fibroids before and after pregnancy and the pregnancy complications were recorded; immunohistochemistry was used to detect the expression of progesterone receptor (PR) and proliferation- and apoptosis-related proteins in the uterine fibroid tissue.

Results: No significant difference was observed in the change in uterine fibroid volume during pregnancy between the treatment group and the control group ( $p>0.05)$. The percentage of uterine fibroids with red degeneration was lower in the treatment group than in the control group, but the difference was not statistically significant. No significant difference was observed in newborn weight, height, Apgar score, threatened miscarriage, or premature birth, among other characteristics, between the two groups ( $p>0.05$ ). Immunohistochemistry showed no significant difference in the expression of $\mathrm{PR}$, cyclinD1, insulin-like growth factor (IGF1), or B-cell lymphoma 2 (Bcl2) between the two groups.
\end{abstract}

Conclusions: The use of dydrogesterone during pregnancy has no significant effect on uterine fibroids, pregnancy progression, or pregnancy outcomes in pregnant patients with uterine fibroids.

Key words: pregnancy, uterine fibroids, dydrogesterone, miscarriage

Ginekologia Polska 2017; 88, 12: 679-685

\section{INTRODUCTION}

Most cases of uterine fibroids occur in women of child-bearing age with strong ovarian function; thus, uterine fibroids are closely related to pregnancy. The incidence of pregnancy with uterine fibroids has continued to increase year after year and has attracted a great deal of attention $[1,2]$. Studies have reported that up to $10.7 \%$ of pregnant women have uterine fibroids [3]. However, the relationship between uterine fibroids and adverse pregnancy outcomes is unknown. The effect of uterine fibroids on pregnancy depends on the size and location of the fibroids. Proper treatment of uterine fibroids that cause infertility might, to some extent, affect the outcomes of the next pregnancy.

Uterine fibroids are hormone-dependent. Due to the significant fluctuations in hormone levels during pregnancy, the size and nature of uterine fibroids, of which there are different types and locations, may change. For pregnant women, the growing uterus may cause torsion of pedunculated subserosal fibroids, and uterine fibroids at the uterine isthmus or the posterior lip of the uterine cervix may block the birth canal. Moreover, uterine fibroids may affect uterine contractions and prevent natural placental separation after delivery, 
which may cause postpartum haemorrhage. Our previous study demonstrated that resection of uterine fibroids during caesarean section does not affect the incidence of pregnancy complications such as postpartum haemorrhage [4]. However, pregnant women with uterine fibroids are at a higher risk for perioperative complications, and numerous studies have demonstrated that uterine fibroids are related to pregnancy complications, such as miscarriage, premature birth, premature placental separation, malpresentation, and low newborn weight. The incidence of these complications varies but, nevertheless, has continued to rise year by year [5].

Dydrogesterone (Duphaston) is commonly used to prevent miscarriage in pregnant women with threatened miscarriage before gestational week 20. Clinical studies have shown that Duphaston exhibits effects similar to those of adrenal hormones, oestrogen, and androgens. Studies have demonstrated that the progesterone receptor (PR) is highly expressed in uterine fibroid tissue, whereas progesterone agents cause an elevation in the mitochondrial membrane potential, and ultimately, abnormal cell proliferation [6]. In addition, studies have shown that PR directly activates and acts on relevant signalling pathways to cause cell proliferation in uterine fibroids [7]. Currently, researchers believe that uterine fibroids rely on the synergistic effect of ovarian steroid hormones, oestrogen, and progesterone for their development and progression and that progesterone may play a more critical role than oestrogen. To date, no in-depth studies have been conducted to investigate the specific effect of Duphaston on uterine fibroids.

To investigate the effect of Dydrogesterone on uterine fibroids during pregnancy, pregnancy complications, and pregnancy outcomes in pregnant women with uterine fibroids, we analysed fibroid growth, pregnancy outcomes, and pregnancy complications in pregnant women with uterine fibroids with or without Duphaston treatment to prevent miscarriage. The purpose was to obtain clinical guidance for the use of Duphaston (to prevent miscarriage) in pregnant women with uterine fibroids.

\section{MATERIAL AND METHODS}

This study was approved by the local research Ethics Committee of Shandong Provincial Hospital in Jinan, China.

\section{General information}

In all, 372 pregnant women with uterine fibroids treated at Affiliated Provincial Hospital of Shandong University between November 2013 and June 2015 were included in this study; of these women, 82 required intervention during pregnancy to prevent miscarriage for various reasons, including the following: red degeneration of uterine fibroids per an abdominal B ultrasound scan during preg-
Table 1. Analysis of the pathogeny of pregnant women with uterine fibroids

\begin{tabular}{|l|c|c|}
\hline Pathogeny & N & Percentage (\%) \\
\hline $\begin{array}{l}\text { Red degeneration of uterine fibroids } \\
\text { (diagnosed by abdominal B ultrasound scan) }\end{array}$ & 12 & 14.63 \\
\hline Hypothyroidism & 2 & 2.44 \\
\hline Antiphospholipid antibody syndrome & 1 & 1.22 \\
\hline Severe gestational vomiting & 1 & 1.22 \\
\hline Gestational diabetes & 7 & 8.54 \\
\hline Gestational hypertension & 5 & 6.10 \\
\hline Premature rupture of membranes & 9 & 10.89 \\
\hline Placental abnormalities & 2 & 2.44 \\
\hline Unexplained vaginal bleeding and lower & 43 & 52.44 \\
\hline abdominal pain & 82 & 100 \\
\hline Total
\end{tabular}

nancy ( $\mathrm{n}=12$; usually presenting with severe sudden lower abdominal pain, which may be accompanied by fever and vomiting), hypothyroidism ( $n=2)$, subclinical hypothyroidism during pregnancy examination $(n=2)$, threatened miscarriage due to incompliance with the dose adjustment of levothyroxine tablets during pregnancy, threatened miscarriage due to antiphospholipid antibody syndrome $(n=1)$, severe gestational vomiting $(n=1)$, gestational diabetes $(n=8)$, gestational hypertension $(n=10)$, premature rupture of membranes $(n=9)$, placental abnormalities $(n=2)$, and unexplained vaginal bleeding and lower abdominal pain ( $n=43)$ (Tab. 1).

Among the 372 pregnant women with uterine fibroids, 82 received intervention to prevent miscarriage. Among them, 33 women with indications during the first trimester received oral Duphaston $20 \mathrm{mg} /$ day for 2 months or longer and did not receive other drugs (the treatment group), and 27 were found to have uterine fibroids during the first trimester but did not receive intervention to prevent miscarriage (the control group). Patients in both groups underwent a caesarean section. General clinical information was compared between the two groups, including the gestational age of the current pregnancy, the number of miscarriages, gestational age, body mass index (BMI), and fibroid size during the first trimester. A B ultrasound scan was performed to measure the maximum diameter $(\mathrm{cm})$ of the fibroid during the first trimester. $\mathrm{BMI}=$ weight $(\mathrm{kg}) /$ height $^{2}\left(\mathrm{~m}^{2}\right)$. Maternal weight and height were measured at the time of admission for labour.

\section{Immunohistochemical staining}

For all patients, uterine fibroids were resected during caesarean section. Fibroids were paraffin-embedded and prepared as 5 - $\mu \mathrm{m}$-thick sections for immunohistochemical 
staining to determine the PR, cyclinD1, insulin-like growth factor 1 (IGF1), and B-cell lymphoma 2 (Bcl2) expression in the two groups. Next, the sections were deparaffinized and rehydrated, and $0.5 \%$ Triton X-100 was used to rupture the membranes. Then, warm $0.01 \mathrm{M}$ citrate buffer ( $\mathrm{pH}$ 6.0) was used for antigen retrieval for 15 minutes, and the sections were immersed in $3 \%$ hydrogen peroxide (diluted in methanol) for 20 minutes to inactivate endogenous catalase. Next, $10 \%$ normal goat serum was used to block the sections, and then the primary antibody (1:100) was added for an overnight incubation at $4^{\circ} \mathrm{C}$. IgG (same dilution) was used as the primary antibody for the negative control. After PBS washes ( 3 times $\times 5$ minutes), the sections were incubated with a biotinylated goat anti-rabbit or goat anti-mouse secondary antibody (Wuhan Boster Biological Technology, Ltd.) at a dilution of 1:200 at room temperature for 40 minutes. After DAB staining and haematoxylin counterstaining, the sections were observed and photographed under an Olympus microscope. Five fields $(\times 400)$ were randomly selected to analyse the staining density of the cytoplasm and the nucleus. The final immunohistochemical staining score $(0-12)=$ density value $\times$ percentage score.

\section{Statistical analysis}

A WPS table was used to establish the database, and SPSS20.0 (Statistical Product and Service Solutions 20.0) statistical software was used for the data analysis. Numerical data are expressed as the mean \pm standard deviation (SD) $(\bar{x} \pm s)$; an independent sample t-test was performed for between-group comparisons, and a chi-squared test was performed to compare the incidence between the two groups.

For the immunohistochemistry results, brown particles indicated positive staining, and the semi-quantitative analysis that was performed incorporated the staining intensity and the scope of distribution. The staining intensity was rated as follows: 0 : no staining, 1: light staining, 2: moderate staining, and 3: strong staining. The scope of distribution was rated as follows: 0 : positive percentage of $0 \%, 1: 1 \%$ to $25 \%, 2: 25 \%$ to $50 \%, 3: 51 \%$ to $80 \%$, and $4: 81 \%$ to $100 \%$. The product of these two scores indicated the protein expression level. A two-tailed Student's t-test was performed to analyse the difference in immunohistochemical staining between the two groups. The data are expressed as the mean \pm SD. $P<0.05$ was considered statistically significant.

\section{RESULTS}

\section{General information of the patients}

The general clinical information, including the gestational age of the current pregnancy, the number of miscarriages, gestational age, BMI, and fibroid size during the first trimester, was compared between the treatment
Table 2. General clinical information of the patients treated with Dydrogesterone and those who were not

\begin{tabular}{|c|c|c|c|}
\hline General information & $\begin{array}{l}\text { Treatment } \\
\text { subjects } \\
(n=33, \bar{x} \pm s)\end{array}$ & $\begin{array}{c}\text { Control } \\
\text { subjects } \\
(n=27, \bar{x} \pm s)\end{array}$ & $\mathbf{p}$ \\
\hline Gestational age & $32.40 \pm 4.11$ & $32.74 \pm 4.74$ & $>0.05$ \\
\hline $\begin{array}{l}\text { Number of } \\
\text { miscarriages }\end{array}$ & $1.00 \pm 1.27$ & $0.78 \pm 0.84$ & $>0.05$ \\
\hline Gestational week & $37.82 \pm 2.68$ & $38.47 \pm 1.47$ & $>0.05$ \\
\hline BMI $\left[\mathrm{kg} / \mathrm{m}^{2}\right]$ & $29.08 \pm 3.24$ & $28.62 \pm 2.72$ & $>0.05$ \\
\hline $\begin{array}{l}\text { Fibroid size during the } \\
\text { first trimester }[\mathrm{cm}]\end{array}$ & $3.44 \pm 2.70$ & $3.37 \pm 2.65$ & $>0.05$ \\
\hline
\end{tabular}

group ( $n=32)$ and the control group $(n=27)$. No significant differences were observed (all $p>0.05$ ) (Tab. 2).

\section{Changes in uterine fibroids after caesarean section}

Fibroid size was indicated as the maximum diameter (cm), and $\Delta \Phi$ (diameter variation) was used to indicate the change in fibroid size before and after caesarean section, wherein $\Delta \Phi=$ fibroid diameter at the time of caesarean section - $\mathrm{fi}$ broid diameter during the first trimester. For the treatment group, the mean fibroid diameter was $3.44 \pm 2.70 \mathrm{~cm}$ during the first trimester and $7.11 \pm 2.38 \mathrm{~cm}$ at the time of caesarean section $(\Delta \Phi 1=3.66 \mathrm{~cm})$. For the control group, these values were $3.37 \pm 2.65 \mathrm{~cm}$ and $7.57 \pm 1.35 \mathrm{~cm}$ during the first trimester and at caesarean section, respectively $(\Delta \Phi 2=4.20 \mathrm{~cm})($ Tab. 3). In both groups, the uterine fibroids were significantly larger at the time of caesarean section than during the first trimester (both $p<0.01$ ), but no significant difference was observed in the change in the uterine fibroid volume between the two groups $(p>0.01)$ (Tab. 3).

\section{Effect of Duphaston on the red degeneration of uterine fibroids}

Routine pathological examination showed that the section of degenerated fibroids was red and soft and that the swirl structure had disappeared. Microscopic observation

Table 3. Changes in uterine fibroids during pregnancy

\begin{tabular}{|c|c|c|}
\hline Fibroid size & $\begin{array}{l}\text { Treatment subjects } \\
\qquad(\mathrm{n}=33, \overline{\mathrm{x}} \pm \mathrm{s})\end{array}$ & $\begin{array}{l}\text { Control subjects } \\
(n=27, \bar{x} \pm s)\end{array}$ \\
\hline $\begin{array}{l}\text { Fibroid diameter during } \\
\text { the first trimester }[\mathrm{cm}]\end{array}$ & $3.44 \pm 2.70$ & $3.37 \pm 2.65$ \\
\hline $\begin{array}{l}\text { At the time of } \\
\text { caesarean section }[\mathrm{cm}]\end{array}$ & $7.11 \pm 2.38^{\#}$ & $7.57 \pm 1.35^{\#}$ \\
\hline Size change $[\mathrm{cm}]$ & $3.66 \pm 1.44$ & $4.20 \pm 2.14$ \\
\hline
\end{tabular}

Note: ${ }^{\#} p<0.01$ 
showed tissue oedema, small vein thrombosis in the tumour, vascular dilatation and congestion, extensive bleeding and haemolysis, a low number of muscle cells, and nuclei dissolution and disappearance. Pathological diagnosis revealed that in the treatment group (intervention was given to prevent miscarriage) $(n=33), 20$ patients $(60.61 \%)$ had red degeneration of uterine fibroids, 7 (21.21\%) had no red degeneration, and the remaining patients had other types of degeneration; in the control group ( $n=27), 21$ patients $(77.78 \%)$ had red degeneration of uterine fibroids, $3(11.11 \%)$ had no red degeneration, and the remaining patients had other types of degeneration. The incidence of red degeneration of uterine fibroids was lower in the treatment group than in the control group, but the difference was not statistically significant ( $p>0.05$ ) (Fig. 1).

\section{Pregnancy complications and pregnancy outcomes}

The percentage of premature rupture of membranes and abnormal foetal position was higher in the treatment group than in the control group; the percentage of foetal distress, placenta previa, postpartum haemorrhage, and large foetus was lower in the treatment group than in the control group. The newborn weight, height, and delivery time (in hospital) were larger or longer in the treatment group than in the control group, and the intraoperative blood loss and operative time were lower or shorter, respectively, in the treatment group than in the control group. However, the differences were not statistically significant (all $p>0.05$ )

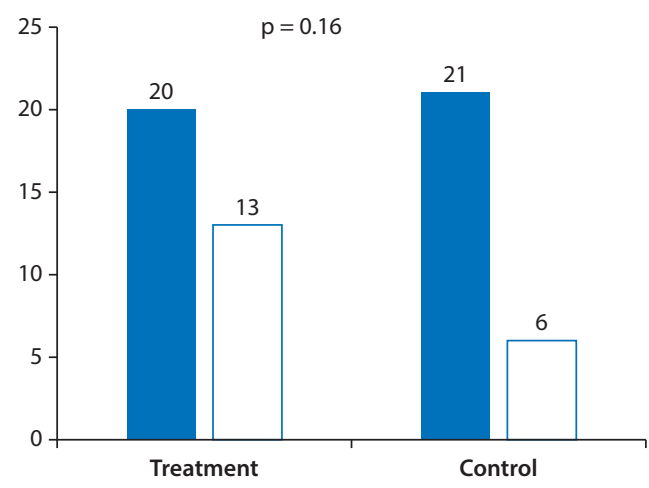

Red degeneration of uterine fibroids $\square$ No red degeneration

Figure 1. Red degeneration of uterine fibroids in pregnancy. Note: In the treatment group (dydrogesterone was given to prevent miscarriage), 20 patients (60.61\%) had red degeneration of uterine fibroids, and 13 had no red degeneration; in the control group, 21 patients had red degeneration of uterine fibroids, and 7 had no red degeneration. The incidence of red degeneration of uterine fibroids was lower in the treatment group than in the control group, but the difference was not statistically significant $(p=0.16)$

(Tab. 4a, 4b). No premature birth or foetal abnormalities were observed in the two groups.

\section{Expression of PR and proliferation-} and apoptosis-related proteins in uterine fibroid tissue obtained from pregnant women during caesarean section

We first performed immunohistochemical staining to evaluate the PR expression in uterine fibroid cells. Figu-

Table 4a. Pregnancy complications

\begin{tabular}{|c|c|c|c|c|c|}
\hline \multirow{2}{*}{ Complications } & \multicolumn{2}{|c|}{ Treatment subjects (total 33) } & \multicolumn{2}{|c|}{ Control subjects (total 27) } & \multirow{2}{*}{$\mathbf{p}$} \\
\hline & n & $\%$ & n & $\%$ & \\
\hline Premature rupture of membranes & 3 & 9.09 & 2 & 7.41 & $>0.05$ \\
\hline Abnormal foetal position & 2 & 6.06 & 1 & 3.70 & $>0.05$ \\
\hline Foetal distress & 1 & 3.03 & 1 & 3.70 & $>0.05$ \\
\hline Placenta previa & 1 & 3.03 & 1 & 3.70 & $>0.05$ \\
\hline Postpartum haemorrhage & 1 & 3.03 & 1 & 3.70 & $>0.05$ \\
\hline Large foetus & 1 & 3.03 & 2 & 7.41 & $>0.05$ \\
\hline
\end{tabular}

\begin{tabular}{|l|c|c|}
\hline Table 4b. Pregnancy outcomes & & \\
\hline Outcomes & Treatment subjects $(\mathbf{n}=\mathbf{3 3})$ & Control subjects $(\mathbf{n}=\mathbf{2 7})$ \\
\hline Blood loss [mL] & $271.52 \pm 124.98$ & $324.07 \pm 105.95$ \\
\hline Operative time [min] & $121.66 \pm 37.62$ & $127.59 \pm 30.17$ \\
\hline Time in hospital (days) & $6.48 \pm 3.21$ & $5.48 \pm 1.09$ \\
\hline Apgar score (score) & $10.00 \pm 0.00$ & $10.00 \pm 0.00$ \\
\hline Newborn height [cm] & $48.24 \pm 1.90$ & $48.04 \pm 3.06$ \\
\hline Newborn weight [g] & $3480.76 \pm 461.38$ & $3309.37 \pm 531.74$ \\
\hline
\end{tabular}




\begin{tabular}{|c|c|c|c|c|c|c|}
\hline \multirow{2}{*}{ Tissue } & \multirow{2}{*}{$\mathbf{n}$} & \multicolumn{4}{|c|}{ PR } & \multirow{2}{*}{$\mathbf{p}$} \\
\hline & & + & ++ & +++ & PR expression detected (\%) & \\
\hline Study subjects & 32 & 3 & 10 & 15 & 87.5 & \multirow{2}{*}{0.488} \\
\hline Control subjects & 27 & 5 & 7 & 9 & 77.8 & \\
\hline
\end{tabular}

Note: PR expression was found in $87.5 \%$ of patients in the treatment group, but the difference between the two groups was not statistically significant ( $p>0.05$ )

A

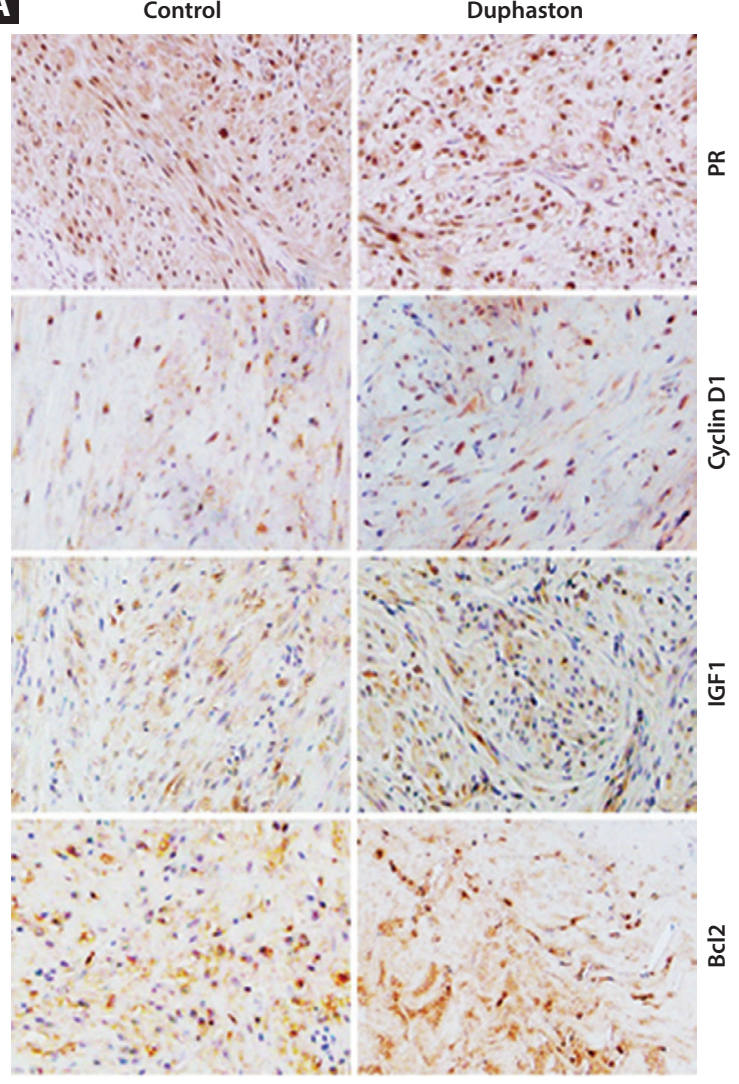

B

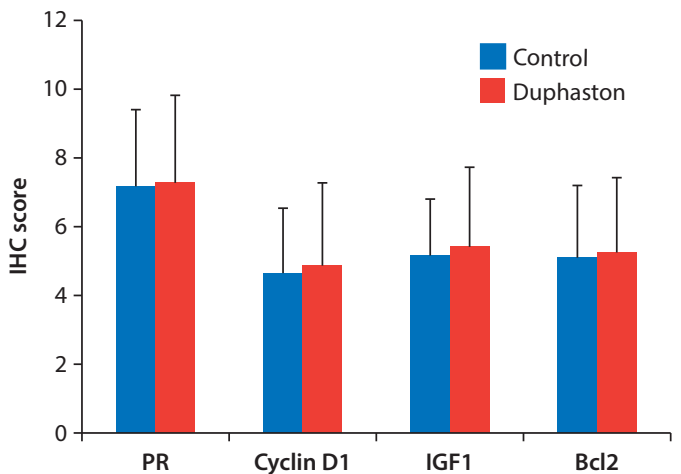

Figure 2. Expression of PR and proliferation- and apoptosis-related proteins in the control group and the treatment (Duphaston) group Note: In this study, we performed immunohistochemical staining to evaluate the expression of PR, cyclinD1, IGF1, and Bcl2 in the control and treatment groups. The results showed no significant difference in protein expression between the two groups re 2 shows no significant difference in PR expression in the nucleus between the treatment group and the control group, which indicates that Duphaston had no effect on PR expression in the nucleus. PR expression was found in $87.5 \%$ of patients in the treatment group, which was higher than the percentage in the control group (77.8\%), but the difference was not statistically significant $(p>0.05)$ (Tab. 5).

We examined the expression of the proliferation-related genes cyclinD1 and IGF1. Duphaston had no significant effect on cyclinD1 or IGF1 expression, which was consistent with our clinical experience (no significant increase in fibroid size was observed after Duphaston treatment).

We determined the $\mathrm{BCl} 2$ expression in the treatment (Duphaston) group and the control group but observed no significant difference between the two groups (Fig. 2).

\section{DISCUSSION}

\section{Progesterone and uterine fibroids}

Numerous clinical studies and experiments have shown that uterine fibroids are hormone-dependent. Recent studies have shown that progesterone promotes uterine fibroid proliferation and inhibits apoptosis, thereby promoting fibroid growth [8]. Oestrogen and progesterone promote fibroid growth in different ways. During pregnancy, the level of maternal oestrogen and progesterone increases significantly, which provides the appropriate conditions for fibroid growth. Currently, it is unclear whether the use of agents such as progesterone during pregnancy to prevent miscarriage may promote fibroid growth or increase the occurrence of red degeneration in uterine fibroids.

\section{Effect of progesterone on uterine fibroids during pregnancy}

In the past three decades, researchers have debated the effect of pregnancy on uterine fibroid volume. Numerous researchers have conducted relevant research but have reached different conclusions [9].

De ViVo et al. [10] revealed that the volume of uterine fibroids significantly increased during pregnancy. Rosati et al. [11] showed that the uterine fibroid volume increased during the first trimester, did not continue to increase during the second and third trimesters, and decreased after 
birth. Some studies have indicated that during pregnancy, uterine fibroids grew at a rate of $0.667 \mathrm{~mm}$ per week during the first and second trimesters [12]. Benaglia et al. [13] also showed that the uterine fibroid volume increased rapidly and significantly during the first trimester.

Most studies showed that uterine fibroids grew during pregnancy; however, some studies reported that during pregnancy, the uterine fibroid volume decreased or remained unchanged. Ozturk et al. [14] reported no significant change in the volume of uterine fibroids during pregnancy. Neiger et al. [15] conducted a longitudinal study of 137 pregnant women with uterine fibroids and found that very few patients showed an increase in uterine fibroid volume (per ultrasound). Phelan et al. [16] suggested that the level of circulating progesterone was significantly higher during pregnancy, but most pregnant women with uterine fibroids did not show an increase or even a decrease in uterine fibroid volume during pregnancy.

In this study, we compared the general clinical information between the treatment group and the control group. We observed no significant difference between the two groups. This study showed that uterine fibroids were significantly larger at the time of caesarean section (end of third trimester) than during the first trimester in both groups; however, the change in the uterine fibroid volume before and after caesarean section did not significantly differ between the two groups. Therefore, although the volume of uterine fibroids increased significantly during pregnancy, the use of progesterone (to prevent miscarriage) did not significantly increase the uterine fibroid volume in pregnant women.

Progesterone promotes the proliferation of uterine fibroid cells [7]. Our results showed that Duphaston had no significant effect on the expression of cyclinD1 or IGF1. This was consistent with our clinical experience, which showed no significant increase in fibroid size after Duphaston treatment. Moreover, we determined the expression of $\mathrm{Bcl} 2$ in the treatment group (Duphaston) and the control group, and no significant difference was observed between the two groups. This finding contradicted previous reports, which showed that progesterone inhibited apoptosis [8]. This contradiction may be related to the small sample size in this study.

\section{Red degeneration of uterine fibroids during pregnancy}

During pregnancy, uterine fibroids may undergo red degeneration due to the unstable state of the coagulation and fibrinolysis system. Studies in pregnant women with degenerated uterine fibroids showed that high-dose natural progesterone affected the blood vessels, increased the blood flow in fibroids, and relieved the acute abdominal syndrome caused by fibroid degeneration, with an intraope- rative finding of shrinkage or disappearance of the original degenerated fibroids [17]. The present study showed red degeneration in $60.61 \%$ of uterine fibroids in the treatment group (progesterone to prevent miscarriage), which was lower than in the control group, but the difference was not statistically significant. In addition, PR expression was found in $87.5 \%$ of patients in the treatment group, which was higher than the percentage of patients in the control group (77.8\%), but the difference was not statistically significant. This finding may be related to the small sample size in this study; nevertheless, the present study demonstrated that Duphaston treatment did not increase the occurrence of red degeneration of uterine fibroids during pregnancy.

\section{Effect of progesterone on pregnancy complications and pregnancy outcomes}

Progesterone is an important steroid hormone that functions to maintain pregnancy during the first trimester. Studies have shown that progesterone prevents premature birth and helps to prevent miscarriage in pregnant women with a short cervix. However, the mechanism by which progesterone prevents premature birth or prolongs gestation is unknown. The conventional belief is that progesterone prevents miscarriage via the inhibition of preterm contractions of uterine muscle fibres or via the inhibition of the expression of factors involved in uterine contractions in the myometrium, such as prostaglandin, oxytocin receptor, and gap protein [18]. Increasingly, researchers believe that progesterone exerts both immunomodulatory and anti-inflammatory effects [19].

Some researchers believe that progesterone may cause the placenta to adhere too tightly, making it difficult to separate during labour, thus contributing to postpartum haemorrhage. Some meta-analyses found no significant difference in maternal or foetal adverse reactions between the progesterone (to prevent miscarriage) group and the control group [20, 21]. The present study demonstrated that the use of dydrogesterone during the first trimester to prevent miscarriages had no adverse effects on pregnancy complications and pregnancy outcomes.

In summary, this was the first study to investigate the effect of dydrogesterone on uterine fibroids during pregnancy, pregnancy complications, and pregnancy outcomes. We conducted several analyses of clinical and laboratory specimens and demonstrated that dydrogesterone (to prevent miscarriage) had no significant effect on the change in uterine fibroids during pregnancy, pregnancy progression, or pregnancy outcomes in pregnant women with uterine fibroids. These results thus provide a theoretical basis for dydrogesterone treatment (to prevent miscarriage) with far-reaching clinical significance. 


\section{Acknowledgements}

This study was funded by the National Natural Science Foundation of China (81270661, 81100447), the Natural Science Foundation of Shandong Province (ZR2009CQ005, ZR2016HM10, ZR201702180317) and the Jinan science and technology plan (201602161).

\section{REFERENCES}

1. Lam SJ, Best S, Kumar S. The impact of fibroid characteristics on pregnancy outcome. Am J Obstet Gynecol. 2014; 211(4):395.e1-395.e5, doi: 10.1016/j.ajog.2014.03.066, indexed in Pubmed: 24705132.

2. Laughlin SK, Herring AH, Savitz DA, et al. Pregnancy-related fibroid reduction. Fertil Steril. 2010; 94(6): 2421-2423, doi: 10.1016/j.fertnstert.2010.03.035, indexed in Pubmed: 20451187.

3. Ciavattini A, Clemente N, Delli Carpini G, et al. Number and size of uterine fibroids and obstetric outcomes. J Matern Fetal Neonatal Med. 2014: 1476-4954.

4. Mu YL, Wang S, Hao J, et al. Successful pregnancies with uterine leiomyomas and myomectomy at the time of caesarean section. Postgrad Med J. 2011; 87(1031): 601-604, doi: 10.1136/pgmj.2009.089748, indexed in Pubmed: 21659453.

5. Munro MG. Uterine leiomyomas, current concepts: pathogenesis, impact on reproductive health, and medical, procedural, and surgical management. Obstet Gynecol Clin North Am. 2011; 38(4): 703-731, doi: 10.1016/j.ogc.2011.09.006, indexed in Pubmed: 22134018.

6. Feng Q, Crochet JR, Dai Q, et al. Expression of a mitochondrial progesterone receptor ( $\mathrm{PR}-\mathrm{M}$ ) in leiomyomata and association with increased mitochondrial membrane potential. J Clin Endocrinol Metab. 2014; 109(5): 344.

7. Kim JJ, Sefton EC. The role of progesterone signaling in the pathogenesis of uterine leiomyoma. Mol Cell Endocrinol. 2012; 358(2): 223.

8. Talaulikar VS, Manyonda I. Progesterone and progesterone receptor modulators in the management of symptomatic uterine fibroids. Eur J Obstet Gynecol Reprod Biol. 2012; 165(2): 135-140, doi: 10.1016/j. ejogrb.2012.07.023, indexed in Pubmed: 22901974.

9. Patel B, Elguero S, Thakore S, et al. Role of nuclear progesterone receptor isoforms in uterine pathophysiology. Hum Reprod Update. 2015; 21(2): 155-173, doi: 10.1093/humupd/dmu056, indexed in Pubmed: 25406186.
10. De Vivo A, Mancuso A, Giacobbe A, et al. Uterine myomas during pregnancy: a longitudinal sonographic study. Ultrasound Obstet Gynecol. 2011; 37(3): 361-365, doi: 10.1002/uog.8826, indexed in Pubmed: 20922776.

11. Rosati $P$, Exacoustòs $C$, Mancuso $S$. Longitudinal evaluation of uterine myoma growth during pregnancy. A sonographic study. J Ultrasound Med. 1992; 11(10): 511-515, doi: 10.7863/jum.1992.11.10.511, indexed in Pubmed: 1404579.

12. Eze CU, Odumeru EA, Ochie K, et al. Sonographic assessment of pregnancy co-existing with uterine leiomyoma in Owerri, Nigeria. Afr Health Sci. 2013; 13(2): 453-460, doi: 10.4314/ahs.v13i2.36, indexed in Pubmed: 24235949.

13. Benaglia L, Cardellicchio L, Filippi F, et al. The rapid growth of fibroids during early pregnancy. PLoS One. 2014; 9(1): e85933, doi: 10.1371/journal.pone.0085933, indexed in Pubmed: 24465797.

14. Ozturk E, Ugur MG, Kalayci $\mathrm{H}$, et al. Uterine myoma in pregnancy: report of 19 patients. Clin Exp Obstet Gynecol. 2009; 36(3): 182-183, indexed in Pubmed: 19860365.

15. Neiger R, Sonek JD, Croom CS, et al. Pregnancy-related changes in the size of uterine leiomyomas. J Reprod Med. 2006; 51(9):671-674, indexed in Pubmed: 17039693.

16. Phelan JP. Myomas and pregnancy. Obstet Gynecol Clin North Am. 1995; 22(4): 801-805, indexed in Pubmed: 8786883.

17. Micronized progesterone in the treatment of imminent necrosis of a myoma during pregnancy.Ultrasound changes during treatment. Ceska Gynekol. 1999; 64(3): 189-192.

18. Matthews SG, Gibb W, Lye SJ. Endocrine and paracrine regulation of birth at term and preterm. Endocr Rev. 2000; 21(5): 514-550, doi: 10.1210/edrv.21.5.0407, indexed in Pubmed: 11041447.

19. Okabe $\mathrm{H}$, Makino $\mathrm{S}$, Kato $\mathrm{K}$, et al. The effect of progesterone on genes involved in preterm labor. J Reprod Immunol. 2014; 104-105: 80-91, doi: 10.1016/j.jri.2014.03.008, indexed in Pubmed: 24933116.

20. Eke AC, Chalaan T, Shukr G, et al. A systematic review and meta-analysis of progestogen use for maintenance tocolysis after preterm labor in women with intact membranes. Int J Gynaecol Obstet. 2016; 132(1) 11-16, doi: 10.1016/j.ijgo.2015.06.058, indexed in Pubmed: 26489489.

21. Haas DM, Ramsey PS, Haas DM, et al. Progestogen for preventing miscarriage. Cochrane Database Syst Rev. 2008(2): CD003511, doi 10.1002/14651858.CD003511.pub2, indexed in Pubmed: 18425891. 